\title{
Land-Sea Delineation in the Visible Channels of Landsat Thematic Mapper
}

\author{
Harilal B. Menon* \& P.V. Sathe \\ National Institute of Oceanography, Dona-Paula, Panaji-403 004
}

\begin{abstract}
Accurate coastal mapping using satellite imageries in the visible region is difficult because of the poor contrast between the radiance received from land and sea, respectively and also due to the structures present in the coastal region caused by chlorophyll, suspended sediments, etc. A method has been suggested to enhance the utility of visible imageries for precise land-sea delineation and detection of tiny islands and extraneous objects in the sea. This method is based on contrast stretching by principal component transformation of visible channels and the Martin-Taylor mapping of transformed components on a video terminal to produce false colour composites to aid visual interpretation.
\end{abstract}

\section{INTRODUCTION}

Detection of water bodies through passive remote sensing is normally done in infrared channels as water and land are at different temperatures, and water acts like a perfect black body for infrared radiation. In case of other passive channels such as, visible and microwaves, the former offers less contrast between water and land while earth's microwave emission is too feeble for any meaningful identification of small water bodies on land or a tiny island in the ocean. Moreover, the atmospheric window ${ }^{1}$ (10 to 12 micrometres region of thermal infrared radiation) has the maximum earth's emission ${ }^{2}$ but has a very low absorption and scattering. Such imageries, therefore, require little atmospheric correction. However, infrared imageries are not very well-suited for precise land-sea delineation because of their comparatively low resolution. For example, the AVHRR operating on NOAA series of satellites gives a resolution ${ }^{3}$ of $1 \mathrm{~km}$. Small islands, dimensions of which are within one unit resolution 
afforded by the infrared sensors, are more likely to be classified as continuation of surrounding waters. Similar constraints also apply while detecting small extraneous objects such as ships, oil-platforms and other offshore installations in the sea for defence purposes. Satellite imageries in the visible region have a far better resolution by virtue of their higher frequencies ${ }^{4}$. Table 1 lists the resolutions obtainable by some sensors operating in different wavelengths.

Visible radiation however, penetrates water bodies and the back scattered radiation emerging out of water is affected by the presence of suspended sediment load, yellow organic dissolved matter and chlorophyll concentration ${ }^{5}$. This process gives a 'structure' 6 to the otherwise uniform water cover (Fig.1). Different coastal processes such as, discharge from rivers, upwelling, marine biodegradation, pollution, etc. continuously feed coastal waters with organic and inorganic substances

Table 1. Resolutions of some sensors operating in different regions of electromagnetic spectrum

\begin{tabular}{lccr}
\hline Sensors & Region & $\begin{array}{c}\text { Wavelength } \\
\text { (micrometers) }\end{array}$ & Resolution \\
\hline SMMR & Microwaves & $10^{3}$ to $10^{6}$ & $150 \mathrm{~km}$ \\
AVHRR & Thermal infrared & 10.5 to 11.5 & $1 \mathrm{~km}$ \\
TM channel 6 & Thermal infrared & 10.4 to 12.5 & $120 \mathrm{~m}$ \\
Spot channel 3 & Infrared & 0.79 to 0.90 & $20 \mathrm{~m}$ \\
Spot channel 1 & Visible & 0.5 to 0.6 & $10 \mathrm{~m}$ \\
\hline
\end{tabular}

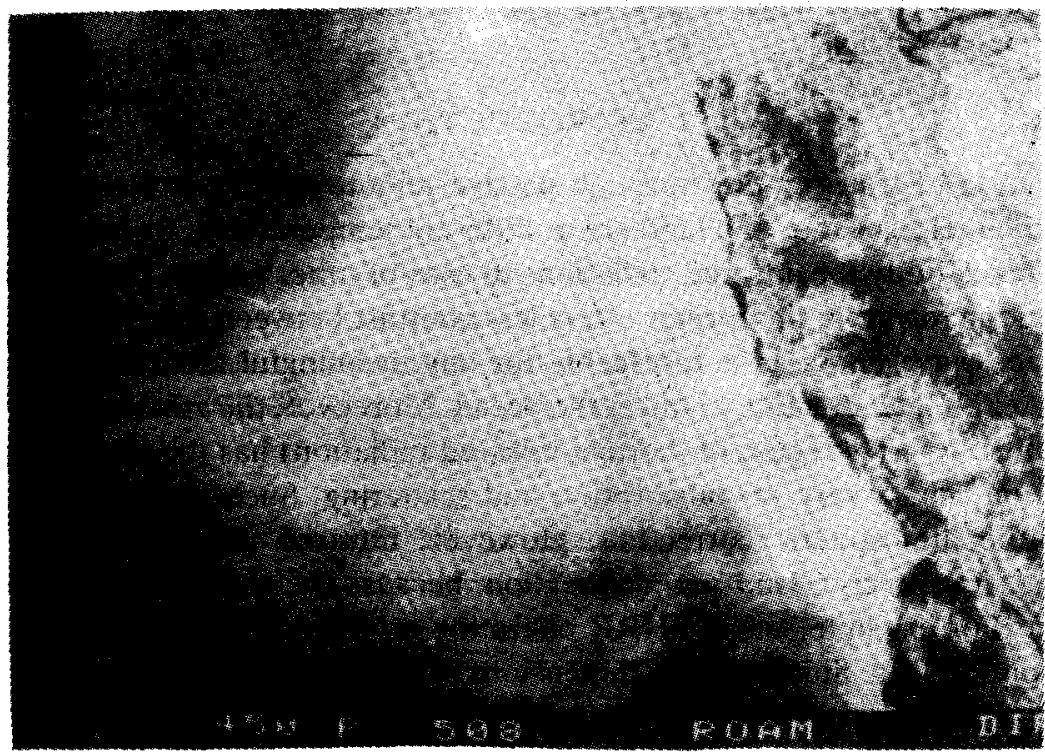

Figure 1. The structure as seen in visible channels in the oceanie region under study. 
contributing to this structure of water as seen in the visible channels. Moreover, the contrast between radiance received from land and from water portions of visible channels is significantly less compared to similar values from infrared channels (Table 2). This makes land-sea delineation in the visible region difficult despite good resolution afforded by it which has been shown in Fig.2. An attempt has been made here to suppress this structure in coastal waters and increase the contrast in the radiance received from land and water portion of the scene. This is done by enhancing the satellite imagery in the visible region. The enhancement is based on principal component transformation (factor analysis) of three visible channels, viz., channels 1 to 3 of Landsat thematic mapper (TM) and the Martin-Taylor mapping of the transformed components on the red-green-blue (RGB) colour triangle to aid visual (false colour) interpretation and also for accurate mapping of coastal zones and tiny

Table 2. Some typical radiance values for land and water cover observed across different portions of the electromagnetic spectrum in a multispectral satellite imagery for a tropical scene*

\begin{tabular}{lccc}
\hline \multirow{2}{*}{ Region } & Wavelength & \multicolumn{2}{c}{ Radiance as 8 bit digital data } \\
Visible & & Land cover & Water cover \\
\hline & $0.52-0.60$ & $60-80$ & $70-85$ \\
Near infrared & $0.63-0.69$ & $25-35$ & $20-35$ \\
Thermal infrared & $1.55-1.75$ & $55-90$ & $10-15$ \\
& $10.4-12.5$ & $70-100$ & $4-6$ \\
\hline
\end{tabular}

* Representative values of the present study for different channels of Landsat TM.

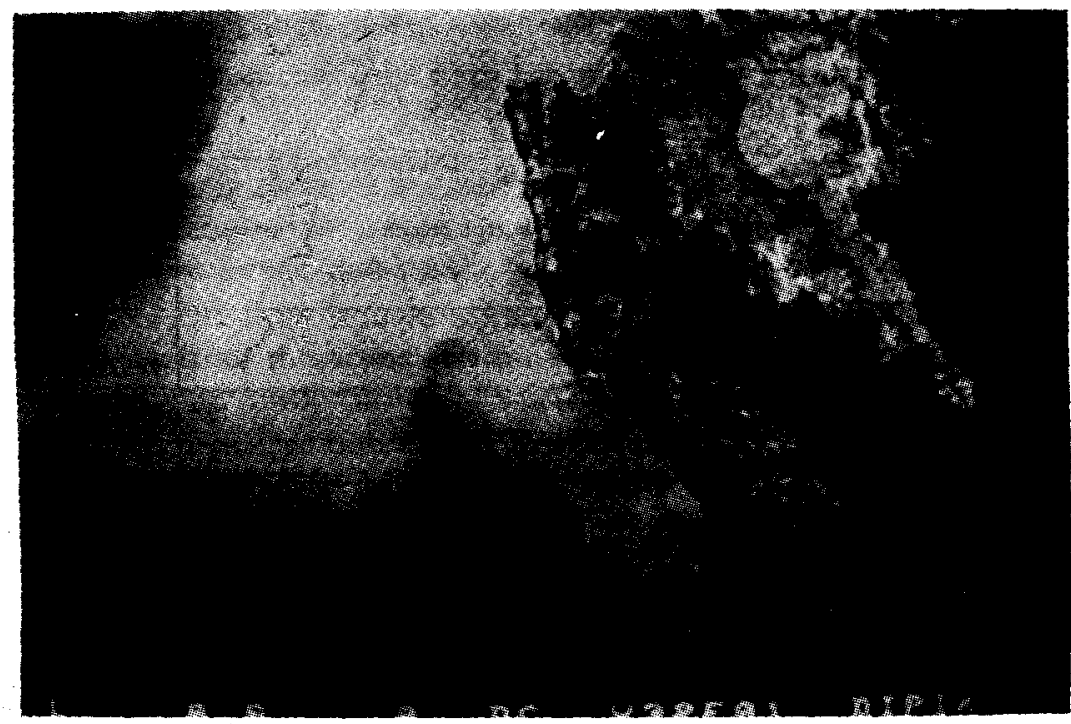

Figure 2. The false colour composite of the first three unenhanced visible channels used in the present study. 
islands. The work was carried out by using a 'Dipix Aries-III Image Analysis System'. This technique can be applied even to aerial photographs to enhance their utility for survey and for defence purposes. Such photographs, however, have to be digitized before enhancement.

\section{MATERIAL}

The first three channels in the visible region of the Landsat thematic mapper CCT (path 148, row 047, quadrant 4) of December 24, 1986 were selected for the present study. The scene covers north Konkan region of the west coast of India which also includes tiny islands called Khanderi and Kolaba Fort (Fig. 3). The scene was corrected for radiometric and earth rotation effects. It contained 2848 lines and 3056 pixels covering geographic areas of approximately $93 \times 88 \mathrm{sq} \mathrm{km}$. This area contains flat lands with and without vegetation, mountainous terrain, coastal sea water with fully developed structure due to suspended sediments and chlorophyll, open ocean with clear water, and tiny islands. Hence, the multidimensional data set of these three visible channels, when loaded on a colour monitor should show significantly different amounts of information. However, it is seen from Fig. 2 that the tiny islands Khanderi and Kolaba Fort are seen almost completely masked by the structure of the surrounding

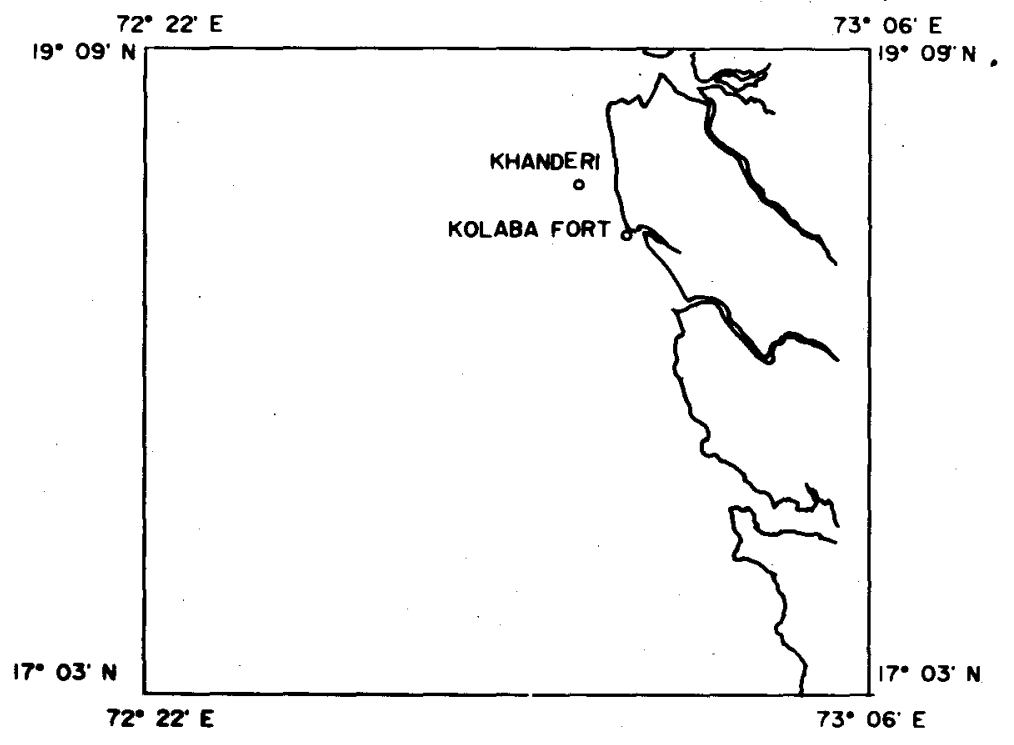

Figure 3. The study area covering north Konkan coast.

waters and the demarcation of land and sea in the coastal region is not precise. The poor contrast between radiance received from land and sea also contributes to this situation. This is usually the case for any imagery in the visible region. Table 3 reflects their statistical parameters. It is seen that all histograms are narrow and peaked. From Table 3 it is also seen that standard deviation and variance of these data sets are small. The minimum and maximum radiance values show that their domains cover only a small portion (not exceeding 1/3) of the entire range from 0 to 255 . The 
Table 3. Statistical parameters of the first three channels of Landsat TM

\begin{tabular}{|c|c|c|c|c|c|c|}
\hline \multirow[t]{2}{*}{ Channel } & \multirow{2}{*}{$\begin{array}{l}\text { Wavelength } \\
\text { (microns) }\end{array}$} & \multirow{2}{*}{$\begin{array}{l}\text { Mean } \\
\text { radiance }\end{array}$} & \multirow{2}{*}{$\begin{array}{l}\text { Standard } \\
\text { deviation }\end{array}$} & \multirow{2}{*}{$\begin{array}{c}\text { Eigen value } \\
\text { (variance) }\end{array}$} & \multicolumn{2}{|c|}{ Radiance } \\
\hline & & & & & Min & Max \\
\hline 1 & $0.45-0.52$ & 9.67 & 11.9 & 141.52 & 0 & 66 \\
\hline 2 & $0.52-0.60$ & 70.92 & 5.05 & 25.52 & 57 & 115 \\
\hline 3 & $0.63-0.69$ & 26.46 & 5.02 & 25.19 & 19 & 63 \\
\hline
\end{tabular}

scatterograms of channel 1 vs 2 and channel 2 vs 3 give the corresponding correlation coefficients 0.75 and 0.57 respectively. Thus it is seen that these three visible channels constitute a highly correlated data set with a low variance in their individual radiance domains. Hence, any enhancement to be done on these channels must substantially increase the variance of the radiance domain and break any correlation existing between them to resolve the various features contained in the scene.

\section{METHOD}

The common methods of enhancement are band ratioing, density slicing, edge enhancement and contrast stretching. Band ratioing is normally performed on uncorrelated data sets and is of little use in the present context as it is likely to reduce the already inadequate land-sea contrast. Density slicing could be useful if land and water covers of the scene give distinctly different histograms. In visible channels, where radiance domains for land and water overlap each other, a density sliced image would show innumerable coast lines both on water as well as on land. Edge enhancement technique is also unsuitable as it will not suppress the structure present in the coastal waters. A mere contrast stretch again would not remove the correlation existing between the channels and hence the method adopted in the present study uses principal component transformation ${ }^{7}$ of the data set which yields a selective contrast stretch and minimizes the correlation. The method involves three stages.

\subsection{Retrieval of Principal Components}

The principal component transformation is essentially a process of converting all pixel values (in the image data) in to values as measured on an alternate set of coordinate axes ${ }^{8}$. The transformation was done across the 'signatures' of a carefully selected sub-area from the scene (training area), which consisted of mountainous terrain and bare land but no water or vegetation. This was done to ensure that the enhancement was restricted only to the land cover of the scene. If the transformation were done across the common signature of the entire scene (as is the common practice), the overall contrast would certainly increase but the features in the area covered by the sea including its structure would also get enhanced and this would go against the main theme of this study. It may be noted here that what is of interest in this work is the land-sea contrast and not just the overall contrast in the scene. Three signatures of this training area were computed (one for each channel), and principal components for each channel were retrieved by rotation of the image data across the corresponding signatures. The mean radiance of these signatures were $40.93,75.83$ and 34.35 
respectively and their eigen values were $27.72,9.15$ and 3.63 respectively. Table 3 shows a comparison of these parameters with those of the whole scene.

\subsection{Inverse Linear Transformation}

The process of generation of principal components from a digital image results into loss of original colours and their relationship to each other. In order to restore this origional colour relationship, a 'decorrelation stretch' developed at the Jet Propulsion Laboratories, California was applied to the transformed components. This simulates the reversal of rotation of feature data to their principal axes maintaining, however, the enhancement and contrast-stretch acquired earlier. Thus three enhanced data sets, each transformed from one channel in the visible region, the contrast of which was stretched to occupy all possible values between 0 and 255 and the enhancement of which was limited to the dry features on land was acquired. This resulted into compression of features in the area covered by sea so that the whole sea could appear as a uniform background of low radiance against which the land and its features could be brightly distinguished.

\subsection{The Martin-Taylor Mapping of Data}

The standard method of displaying three features of a scene on a colour monitor is to map them to red, green and blue, respectively. All input values combine to fill the RGB colour triangle and one gets a single colour display incorporating data from all three features. This method of mapping leaves little choice for the viewer to control the range of colours and intensities or to de-emphasise one feature in relation to the other. Enhanced features such as the ones generated in the present study require other special mappings in which each axis (gun) represents an independently controlled quantity, such as brightness (intensity), hue (tint of a colour), etc. The Martin-Taylor mapping ${ }^{9}$ used in this study extracts contents for each gun from amongst all the three features and generates three independently controlled parameters to be loaded in red, green and blue guns, respectively. These parameters are brightness for red gun, hue for green gun and saturation for blue gun, respectively. Once having loaded the scene on the colour monitor in this way, the viewer is at liberty to alter the parameter of his choice to improve the quality of the image by trying different combinations to bring out the desired results. This is done through a process known as bit-assignment of each feature which results into redistribution of points within the RGB colour triangle. Further discussion on the theory and mapping of Martin-Taylor parameters lies beyond the scope of this paper.

\section{RESULTS AND DISCUSSION}

Figure 4 shows the display on a colour monitor of the three enhanced components obtained from the first three visible channels of the scene under study mapped by the Martin-Taylor technique. It can be seen that the features in the ocean are completely suppressed showing the entire area covered by water as a uniform background of very little radiance against which the land and its features are brightly exhibited. The tiny islands, Khanderi and Kolaba Fort which were nearly masked by the structure present in the surrounding waters (Figs $1 \& 2$ ) are clearly seen as two bright spots in Fig. 4. 
Their locations in the imagery are shown by arrows. The island Khanderi is zoomed and displayed as a single theme in Fig. 5. Such a clear demarcation of this small island in sea as shown in Fig. 5 cannot be obtained in unenhanced visible channels as both the structures in the surrounding water and the poor contrast between land and sea will mask any clarity along the land-sea interface as seen in Fig. 2. Due to the Martin-Taylor mapping of transformed components in the present study, it is possible to have the information shown in Fig. 4 in different colour schemes obtained by the redistribution of data points in different colour combinations. Figure 6 shows a small section of the enhanced imagery covering islands displayed on a colour monitor. This figure clearly shows the shapes, locations and relative sizes of these islands against the dark background of the sea. One more bright spot scene in this figure between the island Khanderi and the main land is a tiny rocky landmass cropping up from the sea known as Underi. This was not evident when the full imagery was mapped on the colour monitor (Fig. 4). Figure 7 shows the scatterograms of enhanced components ( 1 vs 2 and 2 vs 3 ). On comparing the scatterograms of the enhanced and unenhanced channels it can be seen that the existing correlation between the initial data sets could be broken despite the fact that transformation of data set was done across the signature of a selected area from the scene.

Table 4 shows the statistical parameters of the transformed components. Unlike in the initial data (Table 3) it can be seen that the values of the variance of the three transformed components have substantially increased and the range of the radiance values in the transformed components occupy the entire available domain from 0 to 255. This shows that the contrast stretch has been fully achieved in the work. This is responsible for the clear demarcation of the land-sea interface as seen in Figs. 4 to 6. The values of radiance received from the water cover in unenhanced channels were

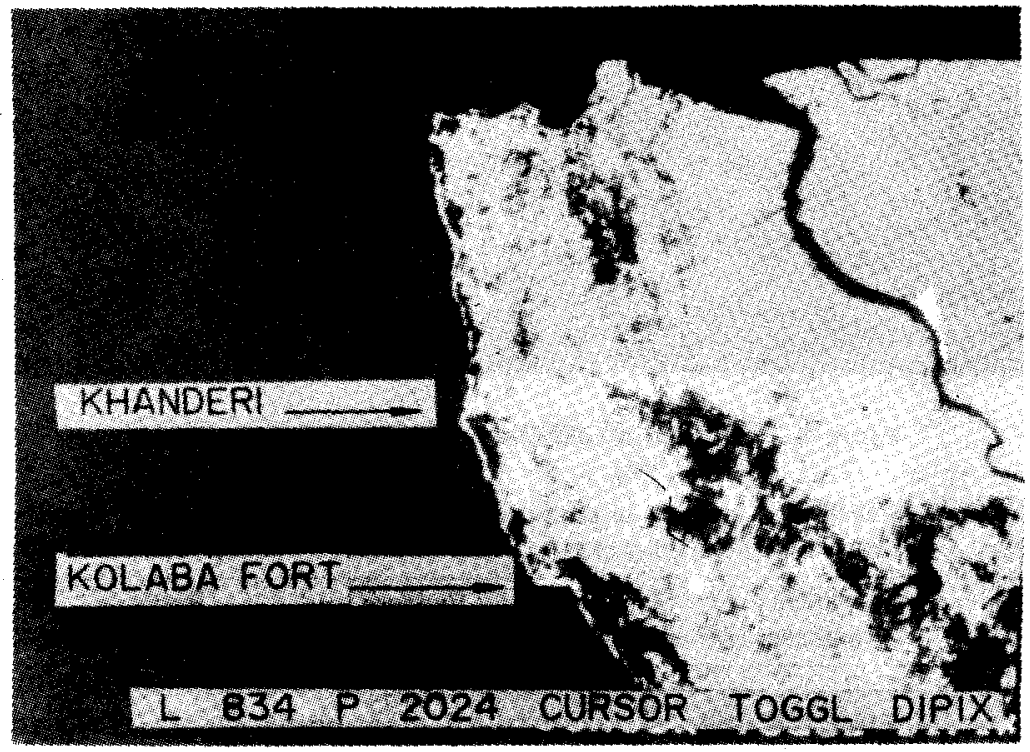

Figure 4. The false colour composite of enhanced components obtained from the first three visible channels of the scene under study. 


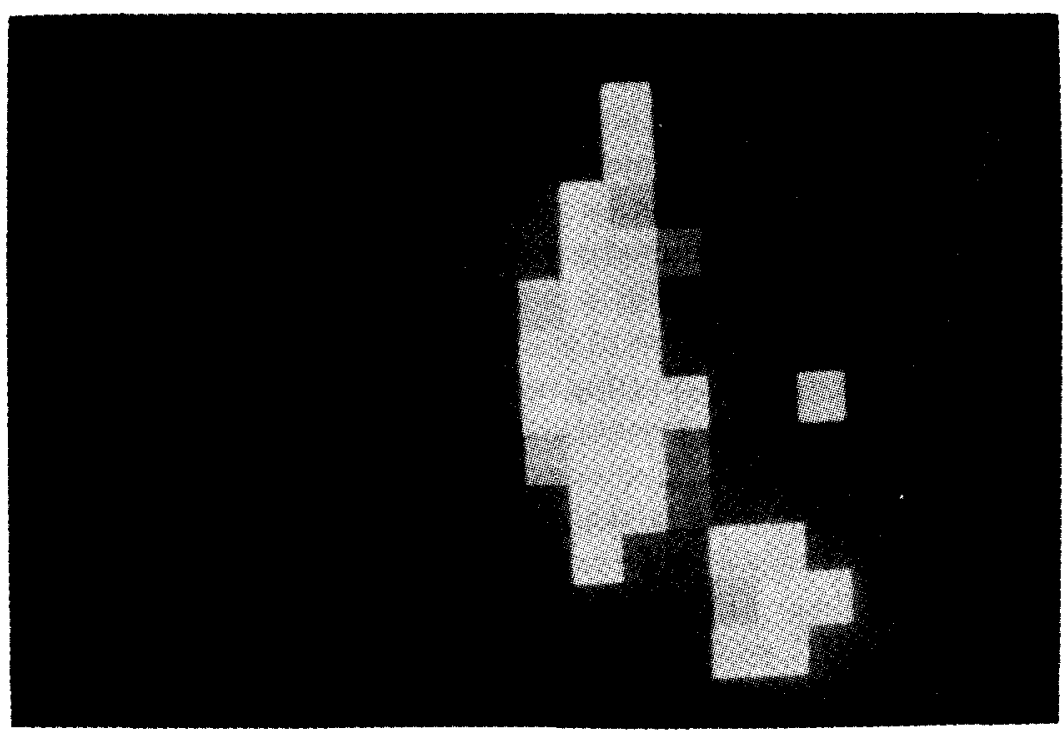

Figure 5. The zoomed image of the tiny island 'Khanderi' after enhancement.

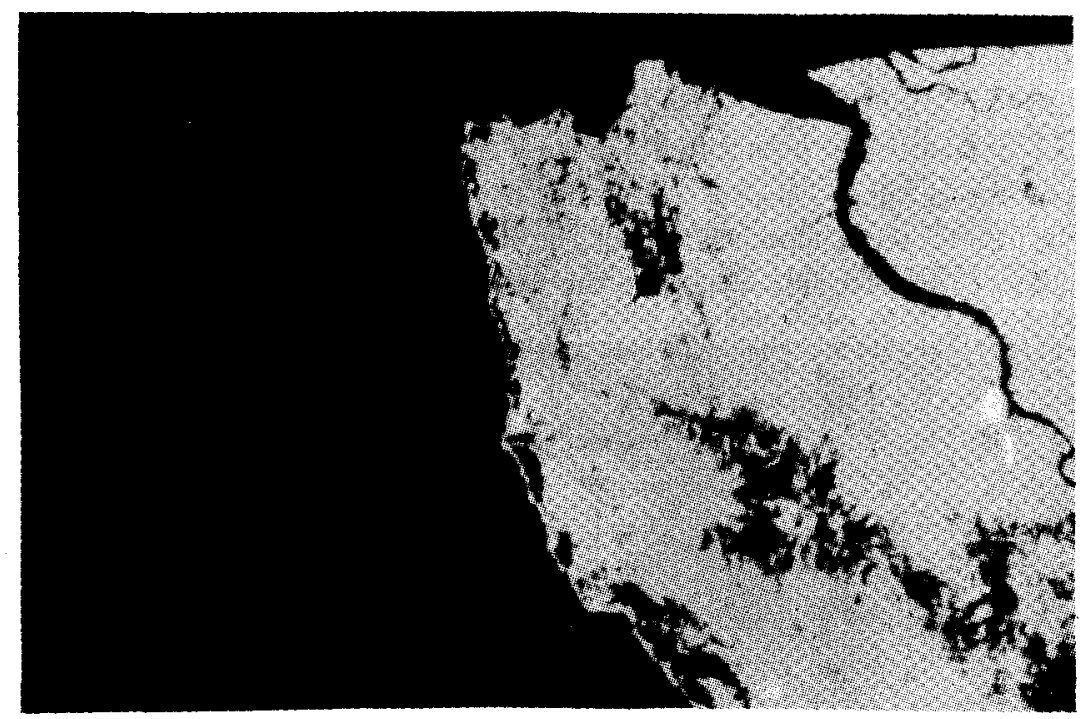

Figure 6. A section of enhanced imagery covering the islands.

Table 4. Statistical parameters of components generated from channels 1,2 and 3 of Landsat TM

\begin{tabular}{lccccc}
\hline Component & $\begin{array}{c}\text { Mean } \\
\text { radiance }\end{array}$ & $\begin{array}{c}\text { Standard } \\
\text { deviation }\end{array}$ & Variance & \multicolumn{2}{c}{ Radiance } \\
1 & 9.38 & 28.28 & 799.75 & 0 & 255 \\
2 & 8.52 & 33.27 & 1106.84 & 0 & 255 \\
3 & 6.14 & 19.76 & 390.65 & 0 & 254 \\
\hline
\end{tabular}




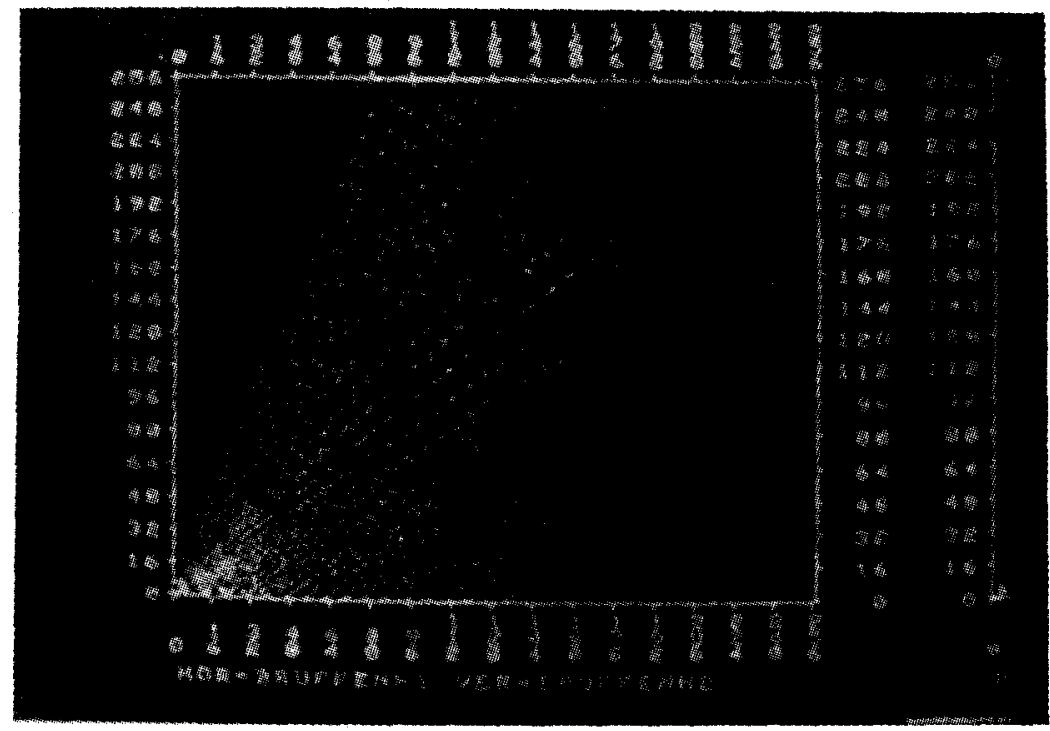

Figure 7 (a). Scatterogram of enhanced components ( 1 vs 2).

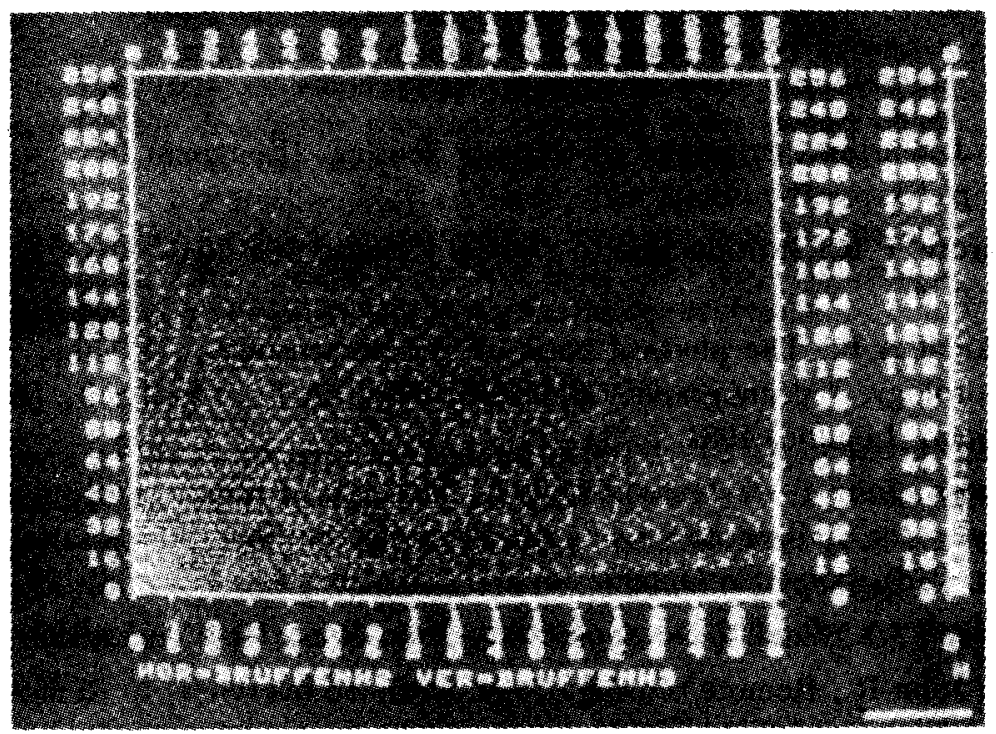

Figure 7 (b). Scatterogram of enhanced components (2 vs 3).

not appreciably different from similar values received from land cover (Table 2), but after the enhancement, the entire area covered by water showed the radiance values in the vicinity of zero while the radiance values received from the land cover were found to have increased considerably. Thus the method of enhancement of a visible imagery by the principal component transformation over the signature of a selected portion of the scene and mapping the components by the Martin-Taylor technique is found to be an efficient way of obtaining the precise land sea-delineation. 


\section{CONCLUSION}

The method of enhancement proposed in this paper greatly increases the utility of visible imageries for identification of water bodies, precise land-sea delineation and spotting of small extraneous objects in the sea at resolutions affordable by visible radiation. Since the structure in the coastal waters is completely suppressed, small islands, ships, offshore installations, etc. present in the open ocean are brightly discernible against uniform and relatively dark cover of the ocean. As aerial photographs suitably digitised can also be subjected to this analysis, their application for defence purposes is greatly enhanced.

\section{ACKNOWLEDGEMENTS}

Authors are thankful to Dr. B.N. Desai, Director, National Institute of Oceanography; Dr. J.S. Sastry, Deputy Director and Head, Physical Oceanography Division and Shri L.V.Gangadhara Rao, Project Coordinator, Remote Sensing Studies, for their encouragement and guidance during the present study. Authors are grateful to the Department of Ocean Development, New Delhi for the funds provided for procuring the Dipix Aries III Image Analysis System without which this study would not have been possible.

\section{REFERENCES}

1. Paul, J. Curran., Principles of Remote Sensing, (Longman Inc., New York), 1985, p. 53.

2. Pisharoty, P.R., Introduction to Optical Remote Sensing, (ISRO, Bangalore), 1977 , p. 4.

3. Cracknell A.P., The physical basis of remote sensing, In Remote Sensing in Meteorology, Oceanography and Hydrology, A.P., Cracknell, (Ed), (Ellis Horwood, London) 1981, p. 36.

4. Johnston, John E. \& Janza, Frank J., The role of remote sensing for energy development, In Remote Sensing Applications for Mineral Exploration, William Smith, (Ed), (Dowden Hutchinson and Ross Inc., Pennsilvania), 1977, p. 199.

5. Robinson, I.S., Satellite Oceanography, (John Wiley, New York), 1985, p. 60.

6. Apel, John R., Remote sensing of the ocean-an overview, In Remote Sensing Applications in Marine Science and Technology, A.P. Cracknell, (Ed), NATO ASI Series C106, 1983, p. 1.

7. Joreskog, K.G., Klovan, J.E. \& Reyment, R.A., Geological Factor Analysis, (Elsevier Scientific Publishing Co., New York), 1976, p. 86.

8. Lillesand, Thomas M., \& Kiefer, Ralph W, Remote Sensing and Image Interpretation, (John Wiley, New York), 198\% p. 469.

9. Aries Image Analysis System, User's Manual, Part II, Chapter 8, Radiometric Corrections, 1986, p. 827. 